\title{
P0144
}

\section{LIGHTING CONTROL USER INTERFACE STANDARDS}

\author{
Bruce Nordman
}

DOI 10.25039/x46.2019.PO144

from

CIE x046:2019

\author{
Proceedings \\ of the \\ 29th CIE SESSION \\ Washington D.C., USA, June 14 - 22, 2019 \\ (DOI 10.25039/x46.2019)
}

The paper has been presented at the 29th CIE Session, Washington D.C., USA, June 14-22, 2019. It has not been peer-reviewed by CIE.

(c) CIE 2019

All rights reserved. Unless otherwise specified, no part of this publication may be reproduced or utilized in any form or by any means, electronic or mechanical, including photocopying and microfilm, without permission in writing from CIE Central Bureau at the address below. Any mention of organizations or products does not imply endorsement by the CIE.

This paper is made available open access for individual use. However, in all other cases all rights are reserved unless explicit permission is sought from and given by the CIE.

CIE Central Bureau

Babenbergerstrasse 9

A-1010 Vienna

Austria

Tel.: +4317143187

e-mail: ciecb@cie.co.at

www.cie.co.at 


\title{
LIGHTING CONTROL USER INTERFACE STANDARDS
}

\author{
Nordman, B. ${ }^{1}$ \\ ${ }^{1}$ Lawrence Berkeley National Laboratory, Berkeley, California, USA \\ bnordman@lbl.gov
}

DOI 10.25039/x46.2019.PO144

\begin{abstract}
For more than a century, the only form of control for most lights was just on or off, controlled from one location using the simplest user interface; usually a push-button or toggle switch. From physical appearance, and placement on a building wall, it was clear that it was a lighting control. Today, light sources can be readily dimmed, have their colour changed, and be responsive to occupancy, daylight, scheduling, and more, with inevitable rising of control complexity. As each manufacturer determines its own conventions for how to communicate concepts to users, the rate at which users correctly understand the controls will drop. A solution to this problem used in other domains, such as vehicle dashboards, is a global language of symbols, supplemented with words, colours, metaphors, physical mappings, and more.

Earlier work determined that no such language for lighting exists. A survey of currently available products found key categories of user interface content: General Principles, Lighting in General, Basic Switching, Brightness, Occupancy Control, Daylight Control, Colour, and Other Topics. This paper reviews considerations for each topic, including select survey findings, and presents elements of a standard for lighting control user interface elements.

While such content should be codified with a recognized standards development organization, manufacturers can use this material in product designs immediately. The language embodied in these symbols and other elements should tightly woven into the fabric of ordinary life. The motivation for this work is to save energy, but other benefits gained from consistent user experience are sufficient to merit a standard.
\end{abstract}

Keywords: Lighting Control, User Interface, Standardization

\section{Introduction}

Almost all uses of energy in buildings actively deliver services to people and have mechanisms to configure or control that process. From temperature setpoints to appliance operation to lighting levels, humans need to occasionally or frequently understand a device's operational state, the potential ways that state could be changed, and how to make changes efficiently and correctly through a user interface.

The most complicated energy-related control system that most people use is that of vehicles, and dashboard symbols and indicators are globally standardized (SAE, 2010). Many controls in a vehicle have energy impacts; others have severe safety implications. Vehicles are shipped globally, and when people travel they often drive, so international consistency is critical.

Some generic user interface principles have been used in lighting: that more is up (at least in many countries) and clockwise, and that basic symbols such as $\mathrm{I}$ and $\bigcirc$ mean on and off, respectively (IEC, 1998; ISO, 2004). However, the lighting industry has lacked a basic structure or set of elements for constructing standard user interfaces.

User interface standardization does not require all controls to be the same; for example, vehicle dashboards vary widely. Rather, interfaces only need to be constructed out of the same vocabulary of individual elements. This is analogous to the way that written text is constructed out of the same set of words (for a given language) but writers still have wide latitude to be creative and compelling in selecting and arranging words. Basic elements in user interfaces are 
words, symbols, colours, metaphors, dynamic behaviour, actuation methods, and audio and tactile interactions; standards can address all of these.

With the present lack of a standard for lighting user interfaces, even manufacturers who desire to make their products more consistently understandable to users cannot do so. When users have difficulty in obtaining the right quantity, quality, and timing of light that they want, energy is likely to be wasted.

Previous work addressed the topic of power control of electronics - turning devices on and off, and most important, making it easier for them to be asleep most of the time (IEEE, 2004; Nordman et al., 2002). That process involved surveying the standards landscape, assessing the user interface elements used in a wide variety of products, understanding the implications of internal system technology, reviewing cultural aspects of some elements, and ultimately proposing content for an international standard.

Some key conclusions of that work were: consistency is more important than intuitive clarity; symbols are preferable to words; and standards and interfaces should embrace simplicity.

\section{Past Research}

Early work (Nordman et al., 2010; Nordman et al., 2011) reviewed relevant standards and literature, and assessed existing lighting control user interfaces. It confirmed that there is no existing standard that covers this topic area specifically, but with an extensive review of related standards, found content relevant to lighting control covering graphical symbols, associations for colour and movement, use of indicators, and terminology.

A more recent review (Nordman et al., 2017; Brown et al., 2019) focused on devices intended for sale in the U.S. Both studies addressed only controls that ordinary people may experience in their work and home life - not interfaces only used in a professional context such as systems for large building control or specialty applications such as theatre lighting ${ }^{*}$. Individual elements were extracted - mostly symbols, but some indicators, words, and more.

This paper includes select findings from this recent review, which relied primarily on information about products from manufacturer web sites. This provides a good record of visual elements such as words and symbols, but detail about indicator light colour and behaviour and dynamic actuation or behaviour are sometimes ambiguous or missing.

A survey of currently available products found key categories of user interface content: General Principles, Lighting in General, Basic Switching, Brightness, Occupancy Control, Daylight Control, Colour, and Other Topics. This paper presents findings from past research, reviews considerations for each topic (including select survey findings), and presents elements of a standard for lighting user interface elements. Some topics have been omitted for space, including speech input, audio output, haptics, ramping/fading during transitions, time-based control, and window shading. See: http://nordman.lbl.gov/lightui.

\section{General Principles}

These general principles are not limited to one of the specific topics that follow and are grouped into overall structure, physical mappings, and use of indicators.

\subsection{Overall Structure - Discussion}

Lighting controls are traditionally placed in prominent locations in a room for convenience, and generally designed to be visually simple and attractive. Most are opaque in exposing details of their capabilities or operation. The number of types of common control modalities is modest and includes toggle/rocker switches, linear sliders, and rotary controls. Many controls indicate status only by their mechanical position, while others use indicator lights.

\footnotetext{
* It would be helpful if professional controls drew on a foundation from ordinary controls, much as truck controls do by starting from the standard automobile symbols but adding many that are only found in specialized vehicles.
} 
Future lighting controls are unlikely to resemble miniature versions of today's vehicle dashboards in which every control is explicitly labelled. That said, the number of control modalities that commonly exist and the portion of controls that have many modalities will increase, so it is likely that significantly more user interface content will be needed.

Some controls have multiple actuation methods accomplished with the same element. These are called secondary actuations. In most cases, there is no indication on the control that the secondary action exists. Secondary actuation mechanisms are typically not obvious to the user without some user instruction and so are confusing if presented without clear visual cues of their existence and associated action. Secondary actuation mechanisms for configuration and maintenance will generally not be employed by ordinary users, and so these functions can be treated differently.

As hardware costs drop, the number of products that include displays or network connections is rising. The number of control abilities present in many light sources has moved past what is reasonable to include in a compact mechanical control.

Standard symbols are references for what a user is expected to have in mind for a concept. A manufacturer may deviate from the reference, but should do so in a way that is readily connectable to the original symbol or concept so that meaning is not lost.

Visual elements may be omitted when meaning remains clear. For example, mechanical switch position can imply on versus off without needing written words or symbols, and controls for lights only (for example, common wall switches) usually do not require a symbol for lighting in general. An indicator light may indicate the status of an on/off or brightness control.

\subsection{Overall Structure - Proposed Standard Content}

Basic use of a lighting control should be clear to a user who has not previously used the control but is familiar with standard user interface elements. Limited experimentation may be needed, such as understanding the light sources to which a control applies.

Lighting controls should have visual cues to indicate that they address lighting. This may be the presence of lighting-specific symbols or visual appearance that is readily associated with lighting control. The location of a control may also be important to how it is perceived.

Lighting concepts and individual elements should not be specific to a particular interface type (e.g. mechanical, display-based, voice-based) or to a particular building type. While individual interface elements are specified, their use is not required. For example, a simple on/off switch may use the mechanical position to indicate which position is on and which position is off, and so not require additional explanation via terms or symbols.

All terms in user interfaces should be appropriately and consistently translated to local language(s). This paper only references terms in English, and does not offer translations. Symbols are preferred over terms, to increase comprehension.

Secondary actuations should be avoided unless there is a graphic indication of their existence and meaning, or if the secondary actuation is for a configuration or maintenance purpose only.

These principles apply to hardware and software for lighting control products, as well as to software configuration and detailed decisions made at the time of product installation.

\subsection{Physical Mappings}

Products sold in the U.S. generally use the normal international conventions (defined in IEC 447) for physical mappings of more and less: more is up, to the right, clockwise, and away (IEC, 1993) ${ }^{\dagger}$. Thus, labelling of which direction is more (or on in the case of a binary control) is not needed, as long as it is clear that on/off or more/less is involved in the control.

\footnotetext{
${ }^{\dagger}$ However, in many other countries, the reverse convention is used, at least for vertical controls, so that up means off.
} 
The standard symbols for plus + (IEC 5005), and minus - (IEC 5006) were created for electrical polarity, as on a battery. Despite this, when used to increase or decrease a value (e.g. brightness) the symbols have clear meaning, particularly when used as a pair.

Lighting controls commonly include a pair of symbols with a triangles pointing up and down, and some pointing left and right. There are no corresponding symbols in international standards; these symbols should be added.

The standard physical mappings of IEC 447 reflect the mappings of the user's action, not the resulting state of the control. For example, a paddle switch pressed in at the top for on will then protrude from the bottom; the action corresponds to up/more, not the resulting position.

\subsection{Physical Mappings - Proposed Standard Content}

Physical mappings of user actions should be used in accordance with IEC 447: An increasing effect is up, clockwise, right, or away; decreasing effect is down, counterclockwise, left, and towards. To indicate controls for more or less (e.g. of light level), the symbols plus + (IEC 5005), and minus - (IEC 5006) may be used. Alternatively, equilateral triangle symbols pointing up or to the right for more, or down or to the left for less may be used.

\subsection{Indicators}

Indicators should not flash in ordinary operation so as not to attract the user's attention. An indicator which calls attention to itself with dynamic behaviour when not warranted is pointlessly distracting and can be annoying.

Some controls have 'locator lights' to make them easier to find in the dark. The survey did not find any controls that labelled such lights, and they were found in various colours, but most commonly white. Locator lights were found on the switch itself, and on an entire group of brightness level indicator lights. In most applications a locator light need not be on when the light is on; some controls turn the locator light off when the light is on. A locator light does not communicate any status information.

\subsection{Indicators - Proposed Standard Content}

A light control may include a 'locator light,' a small light source on a control, which only helps the user find the control when the room or space is dark. Locator lights should be white unless there is a specific reason to be a different colour, but in no case should be red. Locator lights should be constant (not blinking) and can optionally be off when the light being controlled is on.

Indicators should only blink during a dynamic condition (e.g. the lighting control is in a temporary transition state) or the control is seeking to attract the attention of the user.

\section{Lighting in General}

IEC Symbol 5012 (Figure 1) is the most common symbol used on products for the overall concept of lighting in general. It is defined as 'To identify switches which control light sources, e.g. room lighting, lamp of a film projector, dial illumination of a device.' The symbol refers to the control, not to lighting itself. In the product survey most examples of the lighting symbol had seven rays, though some had five, six, or nine. The symbol was found with the base up in some cases and down in others. While controls today vary in the number and length of the rays, shape of the bulb, orientation (pointing up or down), and colour, most are still easily recognizable as related to the standard symbol.

Most controls surveyed did not include a generic symbol for lighting. Manufacturers may assume that users will recognize a lighting control because of its appearance and location; usually the bulb symbol is only present on controls that also include non-lighting functions.

This lamp shape is derivative of incandescent lamps, and so becoming obsolete with the advent of LEDs. However, the key for such symbols is not that people intuit their meaning each time they encounter it, but rather, they learn it once and then recognize it after. 


\subsection{Lighting in General - Proposed Standard Content}

Controls should use IEC symbol 5012, the IEC standard symbol for lighting control (Figure 1), to refer to the overall concept of lighting. An example is to use this symbol to alert a user to the lighting controls section of an application or management system that includes other uses.

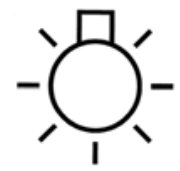

Figure 1 - IEC symbol 5012: Lamp; lighting; illumination

\section{Basic Switching}

Most lighting today is changed through mechanical motion of the user. The ISO 447 mechanical associations are sound and should be followed.

The on $\mathrm{I}$ and off $\bigcirc$ symbols are much more clear when used as a pair. The power symbol can be used to indicate a control that toggles the power state.

\subsection{Basic Switching - Proposed Standard Content}

To switch a light source on or off, controls may use any physical arrangement of control elements as long as they follow prescriptions in ISO 447 for physical mappings. These include that on or more is associated with up, to the right, clockwise, and away. Controls may use the IEC symbols for on I (IEC 5007) and off $\bigcirc$ (IEC 5008), the power symbol $\bigcup_{\text {(IEC 5009), or }}$ words that are the basic translation of these into the local language. Symbols are preferred. Basic switching controls the 'power state' of the light. On and off assign the state; power toggles between the two states.

\section{Brightness}

The basic symbol for variable control, ISO 5004, is defined as 'To identify the control device by means of which a quantity is controlled.' This matches what is needed for controlling the level of light output.

Changing light levels first came into common use with dimming controls, which operate by reducing light output down from a maximum level. Thus, dimming emerged as the organizing metaphor. However, brightness is a better choice as it speaks directly to what is involved rather than a mechanism to change it. Brightness is the noun (the result); the underlying concept of dimming is a verb (the action), the way that one changes the light level. The brightness symbol (Figure 2) is used on many TVs.

There are products on the market that use a large and small version of the brightness symbol, or one with longer and shorter rays, to indicate greater and lower brightness levels. There are products which pair the brightness symbol with the brightness symbol without any rays, which is just a circle - the same as the off symbol.

It seems likely that users would most commonly want a light turned on to return to the most recent brightness level, and while most controls likely operate this way, it is not clear that this should be a required behaviour.

\subsection{Brightness - Proposed Standard Content}

User preferences about adjusting luminance levels should be organized around the concept of brightness, and indicated graphically with IEC symbol 5056, the IEC standard symbol for brightness (Figure 2). 


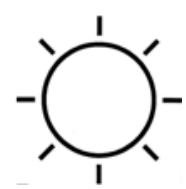

Figure 2 - IEC symbol 5056: Brightness

Brightness may be used to refer to the light output from one or more sources, or to a desired light level at a location which includes light contribution from natural or other artificial sources.

Brightness levels should map onto a numeric (implicitly or explicitly), linear scale to reflect how the user experiences light levels. This may combine the scale produced by a lighting control device with how brightness levels are used by the light source.

The concept of dimming may be used but should be limited to changes in brightness levels. Any standard symbol for variable control may be used (Figure 4 shows four of these).

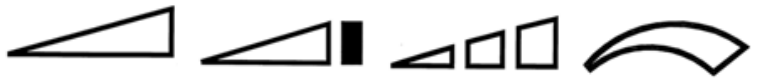

Figure 3 - Standard symbols for Variability: IEC 5004, IEC 5183, IEC 5181, and ISO 1364

As with switching, mechanical associations with brightness levels should follow those specified in ISO 73 / IEC 447, and use of symbols should be as described earlier.

Control for power state and for brightness may be combined.

\section{Occupancy Control}

Occupancy sensors have long been deployed in residential and commercial buildings. When the sensor detects a human in the space, the lights turn on; similarly the lights are shut off when a person is no longer detected. The product survey found no occupancy sensors that have any text or symbols on them to indicate their purpose. Sensors by themselves require no ongoing interaction, but users will commonly want to know that a sensor is involved in controlling light, and understand what sensors are present. Most common today are sensors that combine passive infrared (PIR) and ultrasonic sensing.

Occupancy sensors often have indicators, typically one red and one green LED. Some products use the convention that the indicator for an ultrasonic sensor is green and that for an infrared sensor is red (with the association of red with infrared). The indicators flash when motion is detected so that people in the space can understand when detection is occurring.

As there is no existing international symbol for occupancy, it is informative to assess its current use by manufacturers. Figure 4 shows several marketing symbols for an occupancy sensor. The first, from a display interface, conveys an electromagnetic beacon, that passive infrared $(P I R)$ is the specific mechanism, that people are being detected, and that the light is being turned on and off. The symbols from other manufacturers similarly show people when referencing occupancy sensing.
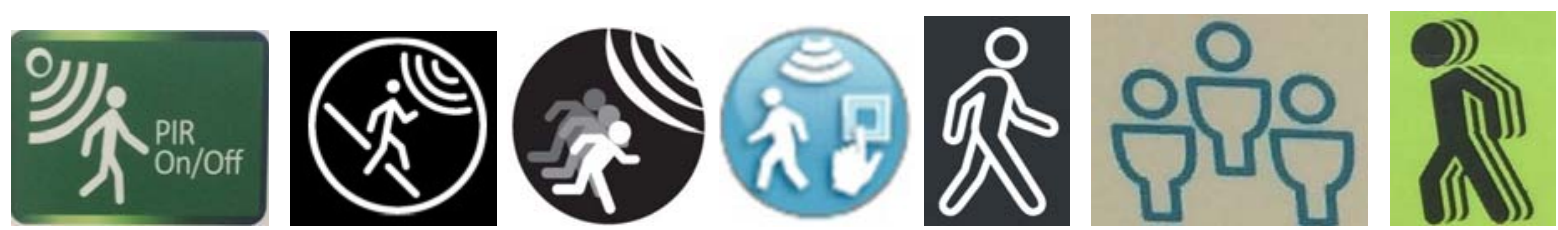

Figure 4 - Occupancy sensing symbols from marketing materials

One potential issue with some of these symbols is that the concentric arcs look very similar to the common Wi-Fi symbol, and so could connote communication rather than sensing. 
Existing symbols for occupancy sensing (found mostly on marketing materials rather than on products themselves) tend to show an abstract person walking along with rays emanating from above. The walking attribute distinguishes these symbols from a stationary person as is used with the restroom symbols. A walking symbol will be distinguished from a running person, which is commonly used as an exit symbol in Europe and elsewhere.

The recommended symbol is the first one in Figure 5 (created by LBNL); it should be added to the international symbol standards. The only advantage of the first symbol over the second is being graphically simpler. It is intended to show a person in a constructed space (room), in a dynamic pose. Traditional occupancy sensors sense movement, but this may not be true of future sensing technologies (e.g. imaging). Symbol designs should include only absolutely essential graphic content.

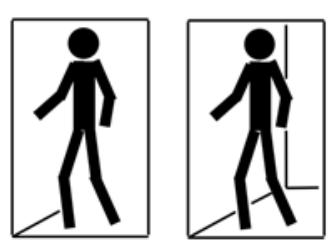

Figure 5 - Occupancy symbols

For indications on occupancy sensors, using red for infrared sensing has an obvious clarity. However, red is supposed to be used only for an error or warning, and so this would require an exception to this general rule. In the long run we may well move onto other technologies; tying this indicator colour to a single technology seems ill-advised. Labelling with a symbol is better.

\subsection{Occupancy Control - Proposed Standard Content}

Light controls that modulate light levels in response to information about human presence in the illuminated space should use the concept of Occupancy. This may include devices that sense occupancy, or controls that act on that information.

To indicate occupancy, controls shall use the symbol shown in Figure 5 (left). This is not an existing ISO/IEC standard symbol, as no such symbol currently exists.

Vacancy control only turns lights off with occupancy sensor information and is a form of occupancy control.

\section{Daylight Control}

A key way to save energy in lighting is dimming (or turning off entirely) artificial light when natural light is available. This requires sensing and actuation, with the term daylight sensor often used (also sometimes ambient light sensor). Similar to occupancy sensors, few if any daylight sensors have a symbol or text on them - they are designed to be minimal and unobtrusive. The survey found some marketing materials with symbols that reference daylight sensing (Figure 6). Several of the images are evocative of the sun, but also are similar to the brightness symbol often used in lighting controls.
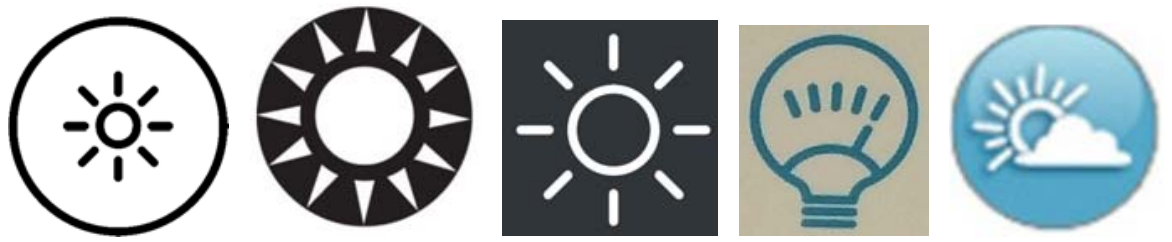

Figure 6 - Light/Daylight sensing symbols from marketing materials

The recommended symbol for daylight sensing or control is shown in Figure 7 (created by LBNL); it should be added to the international symbol standards. The second was considered, but the filled-in circle seems less clearly the sun, though the variable-length rays do convey a 
sense of variability that is helpful. These both adapt existing IEC symbols but add the box to convey a room.

For daylight sensing, technically such systems are sensing and then acting on ambient light, which may be from daylight or from other artificial light sources. However, as the primary application is to reduce energy use of artificial light by taking advantage of freely available daylight, the daylight metaphor is used. The brightness symbol, , has a resemblance to the symbol for natural light, 㳕, but the sun symbol has longer and more rays. The natural light symbol was originally for cameras, not light sources.

\subsection{Daylight Control - Proposed Standard Content}

Light controls that adjust artificial light levels in response to ambient light (principally from daylight) should use the concept of daylight. This may include devices that sense ambient light, or controls that act on that information.

To indicate daylight, controls shall use the symbol shown in Figure 7 (left; created by LBNL). This is not an existing ISO/IEC standard symbol, as no such symbol currently exists; it should be added to the international symbol standards.
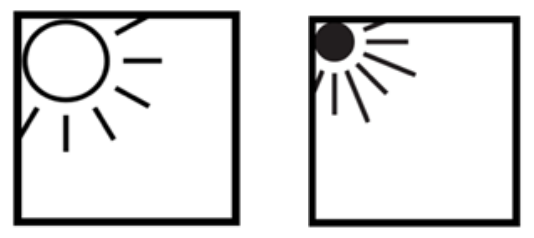

Figure 7 - Daylight symbols

\section{Colour}

More controls are becoming available that allow for fine-grained colour selection.

Many light sources sold today can vary the colour temperature of white light. For colour temperature, the unfortunate fact is that cool white colours are a higher colour temperature than warm whites. Most people do not know this. A correct horizontal scale of colour temperature puts warm at the left and cool at the right (there are lighting controls sold currently that show this). If such 'backwards' colour temperature control were to be deployed widely, it would likely be a significant source of confusion. People do not experience colour temperature in a way at all similar to how they perceive air or water temperature. It would be better to have an alternative metaphor which has the right progression for greater value. It is still early in the deployment of such devices, and so plausible to change the underlying metaphor. After considering alternatives, colour time emerged as the best option.

In the morning the air temperature is relatively cool, often with frost or condensation from breath, and cooler white colours are more common. In the afternoon there is sunset and sometimes in the evening candles or fires, which all convey warm colours. This use of time as the underlying metaphor seems to well match ordinary human experience and is consistent with physical mappings as warmer is then associated with more. The terms cool and warm can still be used - only the explicit reference to temperature is dropped.

Symbols were developed (by LBNL) to convey cool and warm colours with the time-of-day metaphor (see Figure 8); they should be added to the international symbol standards.

\subsection{Colour - Proposed Standard Content}

To indicate the overall concept for light colour, lighting controls that modulate colour should use the IEC symbol for Colour $\bullet$ (IEC 5048). No further concepts or symbols are specified for setting or changing colours.

For colour temperature of white light, the metaphor to use is time-of-day, with 'cooler' white colours associated with the morning, and 'warmer' white colours associated with the afternoon 
or evening. There is no international standard symbol for morning or afternoon, so the symbols to use for cool and warm light are proposed as shown in Figure 8. The arc shows the path of the sun across the sky, with the sun replaced by a lighting symbol.

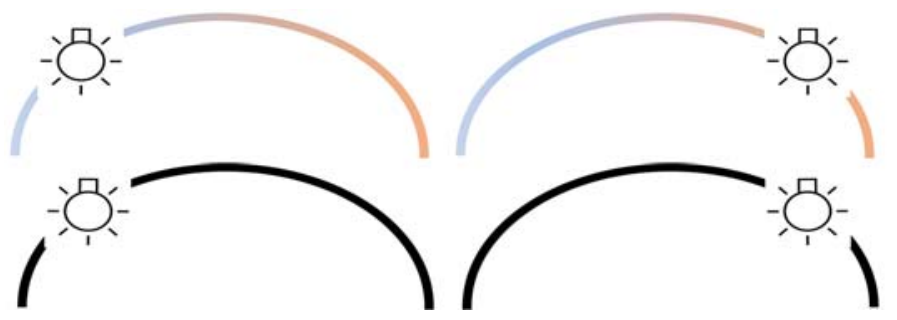

Figure 8 - Symbols for 'Morning White' and 'Afternoon White'

\section{Future Development}

The content above addresses topics that are most important and where good content that can be standardized is apparent. Over time, additional content should be added to standards.

It is important to ensure that the widest range of people can effectively use lighting controls. One dimension of this is to make lighting controls accessible to people with disabilities, such as lack of sight or hearing, as well as to people who are particularly elderly or young. The survey found very little content for accessibility. CIE does address this in the context of traffic signal lights to ensure that they are accessible to people who are colour blind (CIE, 1994).

Some areas, such as colour selection, need more time for experimentation to see what approaches seem to work best. Picking a modest set of content that covers several important areas can best balance benefit and feasibility.

Another issue is making lighting controls work across countries, in the way that vehicle controls do. It is helpful to avoid words when at all possible, when words are used, there should be standard translations for any words needed, including a standard name for each symbol.

Other topics relevant to lighting control are ventilation, networking, configuration, energy saving or consumption tracking, and emerging user interface modalities (e.g. speech, gestures, and haptics). At this time speech seems a particular priority to address, given the increasing use of speech recognition in residential control devices.

Lighting 'scenes' are useful for control and available in many products. They introduce complexity and so likely frequent confusion. However, no clear user interface content on scenes was identified.

Over time we may expect a blurring of distinction between lighting and information displays. Both are now based primarily on the same technology, LEDs. We will increasingly see displays as a source of light, and the ability of lights to modulate intensity and colour, and organize many individual sources, makes them increasingly available to convey information. Use of common concepts and elements between displays and lighting, e.g. brightness, is therefore helpful. These conventions for lighting should therefore be eventually extended to displays.

\section{Summary and Next Steps}

Manufacturers should begin using this content in their design of new products. It should also be adopted by a recognized standards development organization, either international or at national levels. Voluntary or mandatory energy standards could reference the standard in their requirements, though whether compliance should be mandatory needs careful consideration. Most successful user interface standards do not have legal requirements behind them, but rather they gain acceptance because they achieve a critical mass of market penetration and thus consumer 'mind share'.

The goal of this effort is to inform the design of all ordinary lighting controls on the planet, and save energy at no incremental cost. In addition, a lighting standard can serve as an example of 
other domains of energy-relevant controls that could benefit from similar standardization, such as climate control, electricity prices, and generic scheduling and occupancy.

In many countries today, the great majority of lighting controls reverse the IEC 447 convention and use up to mean on (and down to mean off). In some countries both are in wide use. Transitioning to a new scheme is lengthy and not without burden but in the long run preferable. Over time, these countries should shift to consistency with the ISO standard. During the transition, the on and off symbols can be used to clarify how any particular control works.

\section{Acknowledgments}

This project would not be possible without the extensive support of the California Energy Commission. Earlier LBNL work on the project included major contributions from Jessica Granderson, Kelly Cunningham, Margarita Kloss, and Saikiran Dulla.

\section{References}

BROWN, R., P. Schwartz, B. Nordman, J. Shackelford, A. Khandekar, E. Page, N. Jackson, A. Prakash. 2019. Developing Flexible, Networked Lighting Control Systems That Reliably Save Energy in California Buildings. Lawrence Berkeley National Laboratory. California Energy Commission.

CIE Technical Report CIE 107-1994. Review of the official recommendations of the CIE for the colours of signal lights. International Commission on Illumination.

IEC. 1993. Man-machine interface (MMI) - Actuating principles. IEC 447, Second Edition. Geneva, Switzerland: International Electrotechnical Commission.

IEC. 1996. Basic and safety principles for man-machine interface, marking and identification Coding principles for indication devices and actuators. IEC 73. Draft, Geneva, Switzerland: International Electrotechnical Commission.

IEC. 1998. Graphical symbols for use on equipment. IEC 60417. First Edition, Geneva, Switzerland: International Electrotechnical Commission. Part 1: Overview and application; Part 2: Symbol originals.

IEEE. 2004. IEEE 1621, Standard for User Interface Elements in Power Control of Electronic Devices Employed in Office/Consumer Environments.

ISO. 2004. Graphical symbols for use on equipment: Index and synopsis. ISO 7000. Fourth Edition, Geneva, Switzerland: International Organization for Standardization.

NORDMAN, Bruce, and Alan Meier. 2002. The Power Control User Interface Standard. Prepared for California Energy Commission. December.

NORDMAN, Bruce, Jessica Granderson, Alan Meier, Konstantinos Papamichael, Kelly Cunningham and Katherine Wu. 2010. Lighting Controls User Interface Standards. Final Report, prepared for the California Energy Commission / Public Interest Energy Research (PIER) program. October.

NORDMAN, Bruce, Jessica Granderson, and Kelly Cunningham. 2011. Standardization of user interfaces for lighting controls. Computer Standards and Interfaces 34, 273-279. October 10.

NORDMAN, Bruce, Saikiran Dulla, Margarita Kloss. 2017. User Interface Product Survey Report. Lawrence Berkeley National Laboratory. February 10,

SAE 2010. 2010. J2402_201001 - Road Vehicles - Symbols for Controls, Indicators, and TellTales. 